\title{
Special Issue on Deep Learning in Mobile and Wireless Networks: Algorithms, Models and Techniques
}

\author{
Yueshen $X u^{1} \cdot$ Yuyu Yin ${ }^{2} \cdot$ Li Kuang $^{3}$ \\ Accepted: 22 September 2021 / Published online: 8 December 2021 \\ (c) The Author(s), under exclusive licence to Springer Science+Business Media, LLC, part of Springer Nature 2021
}

In recent years, mobile networks and wireless networks have experienced explosive growth. Mobile networks and wireless networks are permeating every aspect of daily lives. As the use of mobile devices and smart applications continues to increase, the demand for mobile applications with deep learning services soars. Inspired by the tremendous success that deep learning has achieved in many tasks on desktop devices, pushing deep learning in mobile networks and wireless networks has become a natural trend. Deep learning has been tried to employed in mobile applications, such as language translation, speech recognition and health monitoring. For many deep learning solutions, the process can be divided into three steps: (1) data collection (2) model training (3) prediction generation. Due to the frequent interaction of mobile devices and wireless devices and users in everyday life, mobile devices and wireless devices collect a large amount of data on users' behaviors, preferences and habits, and thus produce efficient resources for deep learning applications. However, in the training phase, a large amount of data is required to learn the parameters of the model, and to obtain a good model it usually costs a lot of time for training. As a result, most of existing deep learning models are only used for prediction. Due to the limited capacity of mobile devices and wireless devices, the task for applying deep learning in mobile networks and wireless networks is nontrivial.

Yueshen $\mathrm{Xu}$

ysxu@xidian.edu.cn

Yuyu Yin

yinyuyu@hdu.edu.cn

Li Kuang

kuangli@csu.edu.cn

1 Xidian University, Xi'an, China

2 Hangzhou Dianzi University, Hangzhou, China

3 Central South University, Changsha, China
In the paper entitled "Tiny FCOS: A Lightweight Anchorfree Object Detection Algorithm for Mobile Scenarios" by Xiaolong Xu et.al, they design a lightweight FCOS-based model for realtime object detection, named Tiny FCOS, with three distinction characteristics: (1) a lightweight backbone network, which achieves the abatement of model's weight efficiently. (2) a standardized dilated convolution group to construct the structure of FPN and efficiently reduces the impact of the gridding effect. (3) the prediction branch of FCN, while conceptually simple, detect object at only one convolution block rather than stacking convolutions with kernel size of $3 \times 3$ by 4 times.

In the paper entitled "Edge network routing protocol base on target tracking scenario" by Zhongyi Zhang et.al, the authors propose an edge network routing and forwarding protocol based on target tracking scenarios. This protocol can meet the dynamic changes of node locations, and the elastic expansion of node scale. Individual node failures will not affect the overall network, and the network ensures efficient real-time with less communication overhead. The experimental results display that the protocol can effectively reduce the communications volume of the edge network, improve the overall efficiency of the network, and set the optimal sampling period, so as to ensure that the network delay is minimized.

In the paper entitled "Tolerance-Oriented Wi-Fi Advertisement Scheduling: A Near Optimal Study on Accumulative User Interests" by Wanru Xu et.al, the authors study Wi-Fi advertisement scheduling by considering both the tolerance of users in viewing advertisements and resource constraints of Wi-Fi hotspots for broadcasting advertisements. With the goal of maximizing the overall accumulative interests of users, the authors formulate a mixed integer programming problem for Wi-Fi advertisement scheduling. The authors prove that the objective function of this problem is monotone and submodular, subject to a knapsack constraint and a partition matroid constraint. On this basis, the authors solve it by proposing a novel greedy swap algorithm. 
In the paper entitled "Formal Analysis and Automated Validation of Privacy-Preserving AICE Protocol in Mobile Edge Computing" by Jiaqi Yin et.al, the authors propose a new integrated protocol AICE, i.e., authentication and integrity checking on edges, and then formally analyze and automatically validate the correctness and authentication security of the protocol. The authors give the information flows of the AICE protocol by combining the privacy-preserving authentication (PPA) protocol and integrity checking protocol for MEC (ICE) together. According to the features of the AICE protocol, the authors select and extend the SVO logic to conduct the formal analysis of the protocol from the perspective of theoretical analysis of modal logic. Furthermore, the authors employ the AVISPA tool to validate the correctness of the protocol from the perspective of mechanical automatic analysis.

In the paper entitled "SDTIOA: Modeling the Timed Privacy Requirements of IoT Service Composition: A User Interaction Perspective for Automatic Transformation from BPEL to Timed Automata" by Honghao Gao et.al, the authors propose an automatic method of transforming Business Process Execution Language (BPEL) executables into timed automata for formal verification, with the aim of formalizing timed privacy requirements for the IoT service composition and verifying the formal model returned to the UPPAAL supporting tool. First, a privacy requirement template is introduced to analyze the structure of the IoT service composition. Then, a timed computation tree logic (TCTL) property formula template is used to describe the privacy requirements, especially time constraints. Second, an extended timed I/O automata model, namely, the Sensitive Data Timed I/O Automata (SDTIOA) model, is proposed to formalize communication behavior, sensitive data treatment, and service time. Third, the corresponding transformation rules and algorithms are designed for BPEL and SDTIOA.
In the paper entitled "TA-BiLSTM: An Interpretable Topic-Aware Model for Misleading Information Detection in Mobile Social Networks" by Shuyu Chang et.al, the authors propose a novel end-to-end model called Topic-Aware BiLSTM (TA-BiLSTM). TA-BiLSTM could be divided into two modules: a neural topic model and a text classification model. The neural topic model mines latent semantic patterns and encodes relatedness into topic embeddings. The text classification model incorporates latent topic relatedness information into representation by the Topic-Aware attention mechanism and detects misleading information. Experiments on three real datasets show that the proposed TABiLSTM could extract more coherent and semantic topics while enhancing the detecting performance. Furthermore, the authors demonstrate the interpretability of the model via case visualization.

The Guest Editors would like to express their deep gratitude to all the authors who have submitted their valuable contributions, and to the numerous and highly qualified anonymous reviewers. We think that the selected contributions, which represent the current state of the art in the field, will be of great interest to the community. We also would like to thank the Mobile Networks \& Applications publication staff members for their continuous support and dedication. We particularly appreciate the relentless support and encouragement granted to us by Prof. Imrich Chlamtac, the Editor-in-Chief of Mobile Networks \& Applications.

Publisher's note Springer Nature remains neutral with regard to jurisdictional claims in published maps and institutional affiliations. 